\title{
POLIMORFISMOS DE LOS CODONES 136 Y 171 DEL GEN PrPEN UNA MAJADA DE OVINO CRIOLLO DEL URUGUAY
}

\author{
POLYMORPHISMS OF THE 136 AND 171 CODONS IN THE PrP GENE \\ IN URUGUAYAN CREOLE SHEEP
}

\author{
Artigas, R. ${ }^{1 *}$, Passos, D. ${ }^{2}$, Hepp, D. ${ }^{2}$, Weimer, T. ${ }^{2}$ y Postiglioni, A. ${ }^{1}$
}

1Área Genética. Facultad de Veterinaria. UdelaR. Uruguay. *rodyartigas@gmail.com ${ }^{2}$ Laboratorio de Biotecnología. ULBRA. Uruguay.

\author{
Palabras clave adicionales \\ Ovino Criollo. PCR-RFLP. Scrapie.
}

\section{RESUMEN}

El scrapie es una enfermedad neurodegenerativa transmisible de ovinos y caprinos. Se caracteriza por la acumulación en el sistema nervioso central de una isoforma anómala de una proteína celular normal $\left(\mathrm{PrP}^{\mathrm{c}}\right)$ codificada por el gen PrP. En el exón 2 del gen se han identificado mutaciones en los codones 136, 154 y 171. La combinación de estos codones muestran diferentes niveles de asociación con la susceptibilidad a la enfermedad, desde muy resistentes (ARR/ ARR) a muy susceptibles (VRQ/VRQ). Los codones 171 y 136 son los mayores determinantes de la susceptibilidad al scrapie, mientras que el codón 154 juega un rol menor. En el presente trabajo se estudió una muestra de ovinos Criollos Uruguayos $(\mathrm{N}=28)$ para los codones 136 y 171 . Las muestras de ADN genómico se procesaron por PCR-RFLP. Los resultados se obtuvieron por electroforesis en geles de poliacrilamida $(10,5 \%)$ teñidos con $\mathrm{AgNO}_{3}$. Se analizaron las frecuencias genotípicas para los codones 136 y 171. Cinco genotipos diferentes fueron obtenidos, encontrándose en mayor frecuencia los genotipos de alta susceptibilidad: $\mathrm{VQ} / \mathrm{AQ}=0,39$ y $\mathrm{AQ} / \mathrm{AQ}=0,29$. El análisis de riesgo genético de esta majada según los genotipos obtenidos, reveló un $72 \%$ de animales muy susceptibles a la enfermedad en caso de manifestarse las condiciones ambientales propicias.

\section{SUMMARY}

Scrapie is a transmissible neurodegenerative disorder of sheeps and goats. It is characterized by the deposition in the central nervous system of

Recibido: 5-10-09. Aceptado: 20-1-10.

\author{
AdDitional KEYWORDS \\ Creole sheep. PCR-RFLP. Scrapie.
}

an abnormal isoform of a normal cellular protein $\left(\mathrm{PrP}^{\mathrm{c}}\right)$, coded by the PrP gene. Mutations at 136, 154 and 171 codons of exon 2, are described. Combinations of these codons show different levels of association with the susceptibility to this disease; from highly resistants (ARR/ARR) to highly susceptibles (VRQ/VRQ). Codons 171 and 136 are the main determinant of scrapie susceptibility while codon 154 plays a minor role. In the present communication, a sample of Uruguayan Creole sheeps $(\mathrm{N}=28)$ was analysed for 136 and 171 codons. Samples of genomic DNA were processed by PCR-RFLP. The results were obtained by electrophoresis in polyacrylamide gel $(10.5 \%)$ stained with $\mathrm{AgNO}_{3}$. Genotypic frequencies for the 136 and 171 codons were analysed. The results revealed 5 different genotypes. The most frequent genotypes were those associated with more susceptibility to the disease: $V Q / A Q=$ 0.39 y $A Q / A Q=0.29$. The analysis of genetic risk revealed a $72 \%$ of highly susceptible individuals, in case of proper environmental conditions.

\section{INTRODUCCIÓN}

El scrapie es una enfermedad neurodegenerativa transmisible de ovinos y caprinos. Cambios degenerativos (de aspecto espongiforme) en el cerebro, son los responsables de la sintomatología nerviosa y de la muerte inevitable de los animales infectados. La enfermedad se caracteriza por la acumulación de amiloides en el siste- 
ma nervioso central y retículo endotelial, de una isoforma anómala $\left(\mathrm{PrP}^{\mathrm{Sc}}\right)$ de la proteína prión celular $\left(\mathrm{PrP}^{\mathrm{c}}\right)$, codificada por el gen $\operatorname{PrP}$.

Mutaciones en los codones 136, 154 y 171 se han asociado en numerosas razas, a grados variables de resistencia a la enfermedad y a la edad en que la misma se manifiesta en los animales portadores (Hunter, 1997). El codón 171 (con los alelos: $\mathrm{R}=$ arginina, $\mathrm{Q}=$ glutamina, $\mathrm{H}=$ histidina o $\mathrm{K}=$ lisina) es el mayor determinante de la susceptibilidad al scrapie, siendo los alelos R y Q los utilizados para control de la enfermedad (Passos, et al., 2008). El codón 136 (con las variantes: $\mathrm{A}=$ alanina o $\mathrm{V}=$ valina) también afecta la susceptibilidad para algunos tipos de scrapie (Goldmann, 2008), mientras que el codón 154 (con los alelos $\mathrm{R}$ y H) juega un rol menor (Hunter et al., 2002).

Dentro del codón 136, los alelos A y V se asocian respectivamente, con resistencia o susceptibilidad al scrapie mientras que en el codón 171, el alelo resistente corresponde al R y el susceptible al Q. De esta manera, el genotipo AR/AR confiere elevada resistencia, mientras que los individuos VQ/VQ son altamente susceptibles (YuzbasiyanGurkan et al., 1999). La existencia de factores poligénicos y ambientales asociados también con la manifestación de la enfermedad deben ser considerados (Díaz et al., 2005).

En Uruguay se ha identificado una majada de ovinos Criollos en el Parque Nacional de San Miguel (Depto. Rocha) no sujeta a programas de selección, por lo que su variabilidad genética es principalmente debida a efectos de la selección natural. Estos animales habitan la zona este del país, próximos a Brasil (Estado de Rio Grande do Sul), país donde han sido diagnosticados casos clínicos de scrapie (Passos et al., 2008).

En el presente trabajo, se pretende brindar información preliminar acerca de la distribución alélica, genotípica y haplotípica de los principales codones 136 (A, V) y 171 $(\mathrm{R}, \mathrm{Q})$ del gen $\operatorname{Pr} P$, en ovinos Criollos Uruguayos.

\section{MATERIAL Y MÉTODOS}

Se estudiaron 28 ovinos Criollos Uruguayos de ambos sexos pertenecientes al Parque Nacional de San Miguel (Depto. de Rocha; $33^{\circ} 40^{\prime} \mathrm{S}$ y $\left.53^{\circ} 38^{\prime} \mathrm{W}\right)$. Dentro del muestreo fueron incluidos la totalidad de los carneros de la majada $(\mathrm{N}=13)$, que actualmente cuenta con 300 animales. Las muestras de sangre fueron extraídas con anticoagulante (EDTA) a partir de vena yugular y conservadas a $-20^{\circ} \mathrm{C}$ hasta su procesamiento. El ADN genómico fue aislado por el método convencional de fenolcloroformo y posterior precipitación con etanol. La elección de los primers, PCR y condiciones de restricción, fueron las descritas por Yuzbasiyan-Gurkan et al. (1999). Los productos de PCR fueron analizados por electroforesis vertical en geles de poliacrilamida no desnaturalizante al 10,5\% y teñidos con $\mathrm{AgNO}_{3}$, utilizando marcadores de peso molecular de $25 \mathrm{bp}$ y $10 \mathrm{bp}$. Las frecuencias alélicas y haplotípicas fueron estimadas mediante conteo directo de alelos, utilizando el programa de genética poblacional GENEPOP32 V1.31 (Raymond y Rousset, 1995).

\section{RESULTADOS}

La amplificación para los codones $136 \mathrm{y}$ 171 mostró amplicones de 266 y 159 pb. respectivamente. La digestión de estos productos reveló para el codón 136 fragmentos de restricción de 176,60 y 30 bp para el alelo A y de 206 y 60 bp para el alelo V (figura 1A). En el codón 171 los fragmentos observados fueron de 81, 60 y $18 \mathrm{bp}$ para el alelo $\mathrm{R}$ y de 99 y 60 bp para el alelo Q (figura 1B), según lo descripto por Yuzbasiyan-Gurkan et al. (1999).

Las frecuencias alélicas, genotípicas y haplotípicas se muestran en la tabla I. Los genotipos más frecuentes fueron AA $(57 \%)$ y QQ $(65 \%)$, no observándose el genotipo VV.

El haplotipo más común fue el AQ, sien- 
Tabla I. Frecuencias genotipicas, alélicas y haplotípicas para los codones PrP 136 y 171. (Genotype, allelic and haplotype frequencies for codons PrP136 and 171).

\begin{tabular}{lc}
\hline Genotipo de PrP & Frecuencias \\
\hline Codón 136 & \\
AA & 0,57 \\
A V & 0,43 \\
Frecuencias alélicas: & \\
A & 0,79 \\
V & 0,21 \\
Codón 171: & \\
RR & 0,14 \\
QR & 0,21 \\
QQ & 0,65 \\
Frecuencias alélicas: & \\
R & 0,25 \\
Q & 0,75 \\
Frecuencias haplotípicas: & \\
AR & 0,23 \\
AQ & 0,55 \\
VQ & 0,22 \\
\hline
\end{tabular}

do la frecuencia de los haplotipos AR y VQ muy similar (tabla I).

Dentro de la combinación haplotípica, el genotipo $\mathrm{VQ} / \mathrm{AQ}$ fue el más frecuente, seguido por el genotipo AQ/AQ (tabla II).

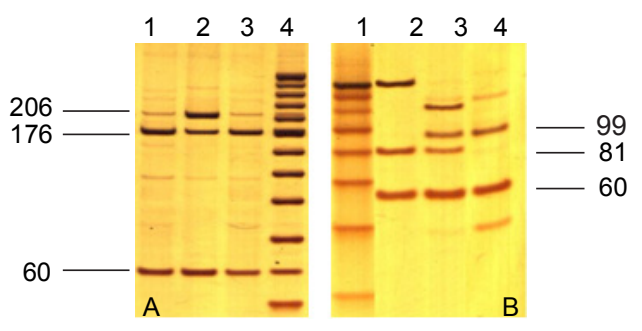

A) codón 136: carriles 1 y 3 genotipo AA; carril 2 genotipo VA; carril 4 marcador de peso molecular 25 bp. B) carril 1 marcador de peso molecular 10 $\mathrm{bp}$; carril 2 genotipo RR; carril 3 genotipo QR; carril 4 genotipo QQ.

Figura 1. Producto de digestión de los codones 136y 171. (Digestion products of codons 136 and 171).
Tabla II. Frecuencia genotípica para los codones PrP 136/171 y clasificación en grupos de riesgo. (Genotype frequency for codons $\operatorname{PrP} 136 / 171$ and genetic risk).

\begin{tabular}{ccc}
\hline Grupo de riesgo* & Genotipo & Frecuencia \\
\hline R1 & AR/AR & 0,14 \\
R3 & AQ/AR & 0,14 \\
R4 & AQ/AQ & 0,29 \\
& AQ/VR & 0,04 \\
R5 & AQ/VQ & 0,39 \\
\hline
\end{tabular}

*Grupos de riesgo de acuerdo a Dawson et al. (1998): R1: bajo riesgo a scrapie; R3: riesgo moderado; R4 y R5: alto riesgo.

\section{DISCUSIÓN}

La caracterización molecular del gen $\operatorname{Pr} P$ en esta majada, reveló para el codón 136 una alta frecuencia del alelo de resistencia $(\mathrm{A}=$ $0,79)$ no observándose en ninguno de los animales analizados la presencia del alelo de hipersensibilidad en estado de homocigosis (VV). Datos similares han sido reportados por Passos et al. (2008) en las razas Suffolk y Hampshire Down de Brasil, no obstante la frecuencia del genotipo AV (tabla I) difiere de la encontrada para las razas anteriores (Suffolk: 0,01 y Hampshire Down: 0,12).

En relación al codón 171, el alelo Q, asociado a mayor susceptibilidad, fue el más frecuente $(75 \%)$, detectándose el alelo de resistencia $\mathrm{R}(25 \%)$ en menor proporción. Relaciones alélicas similares han sido determinadas para otras razas ovinas de la región (Passos et al., 2008; Pinto et al., 2001).

La combinación de los alelos identificados para los codones 136 y 171 da lugar a cuatro haplotipos posibles (AQ; AR; VQ; VR) detectándose 3 en este estudio (tabla I). La frecuencia de los haplotipos AR y AQ en ovino Criollo, no difieren de las descritas en razas locales españolas pertenecientes al mismo tronco etnológico, como Latxa Cara Negra y Latxa Cara Rubia (Sanz-Parra et al., 


\section{ARTIGAS, PASSOS, HEPP, WEIMERY POSTIGLIONI}

2001) y Rasa Aragonesa (Ponz et al., 2003). Sin embargo se encuentran diferencias en la frecuencia del haplotipo hipersensible (VQ), siendo mayor en ovino Criollo Uruguayo $(0,22)$. La alta frecuencia del haplotipo AQ en esta majada (55\%), no sujeta a ningún plan de selección y los valores similares encontrados en otras razas (no seleccionadas por resistencia a la enfermedad) refuerzan la teoría de este haplotipo como el ancestral (Ponz et al., 2003).

Cuando los genotipos obtenidos son categorizados en una escala de riesgo (Dawson et al., 1998), el 72\% de los animales pertenecen a los grupos R4 y R5 de riesgo alto y muy alto a desarrollar la enfermedad.

\section{BIBLIOGRAFÍA}

Dawson, M., Joinville, L.J., Hossie, B.D. and Hunter, N. 1998. Guidance on the use of PrPp genotyping as an aid to the control of clinical scrapie. Vet. Rec., 142: 623-625.

Díaz, C., Vitezica, G.Z., Rupp, R., Andréoletti, O. and Elsenn, J.M. 2005. Polygenic variation and transmission factors involved in the resistance/ susceptibility to scrapie in a Romanov flock. J. Gen. Virol., 86: 849-857.

Goldmann, W. 2008. PrP genetics in ruminant transmissible spongiform encephalopathies. Vet Res., 39: 30.

Hunter, N., Foster, J., Chong, A., McCutcheon, S., Parnham, D., Eaton, S., Mackenzie, C. and Houston, F. 2002. Transmission of prion diseases by blood transfusion. J. Gen. Virol., 83: $2897-$ 2905.

Hunter, N. 1997. PrP genetics in sheep and the implications for scrapie abd BSE. Trends Microbiol., 5: 331-334.

Passos, D.T., Ribeiro, L.A.O., Rodrigues, N.C. Hepp, D. and Weimer, T.A. 2008. PrP polymorphisms in Brazilian sheep. Small Ruminant Res., 74: 130-133.
El elevado porcentaje de animales incluidos en los grupos de mayor riesgo hacen a este gen de importancia al momento de implementar programas de recuperación del ovino Criollo.

En la actualidad se carece de pruebas rápidas que permitan el diagnóstico en la fase subclínica de la enfermedad. Ante esta situación el genotipado adquiere especial importancia como herramienta para la vigilancia epidemiológica. El conocimiento del riesgo genético de cada población, principalmente de aquellas poblaciones fronterizas (como es el caso de estos ovinos Criollos) permitirá implementar más y mejores controles para prevenir la aparición de scrapie.

Pinto, G.B., Jiménez, L.S., Zandomeni, R., Viera, J.B., Carrillo, B.J. y Weber, E.L. 2001. Análisis del polimorfismo del gen PrnP en ovinos de la Argentina. Vet. Arg., 18: 103-109.

Ponz, R., Monteagudo, L.V. y Arruga, M.V. 2003. Estimación de frecuencias genotípicas del gen PrP en la raza ovina rasa Aragonesa. Identificación de animales con alelos sensibles a scrapie. Arch. Zootec., 52: 85-88.

Raymond, M. and Rousset, F. 1995. GENEPOP (version 1.2): Population genetics software for exact tests and ecumenicism. J. Hered., 86: 248-249.

Sanz-Parra, A., Barandika, J., Beltran de Heredia, I., Arrese, F., Hurtado, A., Juste, R.A., Oporto, B., García Crespo, D. y García-Perez, A.L. 2001. Patrón de resistencia genética al scrapie en ganado ovino de raza Latxa. ITEA, 22: 66-68. Yuzbasiyan-Gurkan, GVm, Krehbiel, J.D., Cao, Y. and Venta, P.J. 1999. Development and suefulness of new polymerase chain rectionbased test for detection of different alleles at codons 136 and 171 of the ovine prion protein gene. Am. J. Vet. Res., 60: 884-887. 